\title{
Novedades sobre la sociedad de economía mixta en la Ley de Contratos del Sector Público de 2017
}

\author{
María Dolores Rego Blanco ${ }^{1}$ \\ Universidad Pablo de Olavide \\ mregbla@upo.es
}

\begin{abstract}
NOTA BIOGRÁFICA
M. ${ }^{a}$ Dolores Rego Blanco es Licenciada en Derecho por la Universidad de Sevilla y Doctora en Derecho por la Universidad Pablo de Olavide. Es Profesora Titular de Derecho Administrativo en la Universidad Pablo de Olavide. Sus líneas de investigación abarcan temas de garantías de los ciudadanos frente a las Administraciones Públicas, en el ámbito de la jurisdicción contenciso-administrativo y del procedimiento administrativo, así como de la administración electrónica, y de la contratación pública.
\end{abstract}

\section{SUMARIO}

I. INTRODUCCIÓN: LA IRRUPCIÓN DE LA SOCIEDAD DE ECONOMÍA MIXTA EN LA LEGISLACIÓN CONTRACTUAL. II. DIRECTIVAS EUROPEAS DE 2014 Y SOCIEDAD DE ECONOMÍA MIXTA ¿EFECTO DIRECTO, DEBER DE TRANSPOSICIÓN O LIBERTAD LEGISLATIVA? III. LA DISPOSICIÓN ADICIONAL 22. ${ }^{a}$ DE LA LCSP DE 2017: NOVEDADES Y OPORTUNIDADES PERDIDAS PARA LAS SOCIEDADES DE ECONOMÍA MIXTA. III.1. ¿AÚN EN UNA DISPOSICIÓN ADICIONAL? III.2. LA NUEVA LCSP ALTERA LA DEFINICIÓN LEGAL DE SOCIEDAD DE ECONOMÍA MIXTA. III.3. LA SOCIEDAD DE ECONOMÍA MIXTA PUEDE CREARSE POR CUALQUIER ENTE DEL SECTOR PÚBLICO, NO SOLO POR ADMINISTRACIONES PÚBLICAS. III.4. LA FÓRMULA DE COLABORACIÓN PÚBLICO-PRIVADA DE ECONOMÍA MIXTA SIGUE RESTRINGIDA A «SOCIEDADES». III.5. VARIACIONES SOBRE EL OBJETO DE LA SOCIEDAD DE ECONOMÍA MIXTA: SE DEJAN FUERA LOS ANTIGUOS CONTRATOS DE GESTIÓN DE SERVICIOS PÚBLICOS QUE AHORA SON CONTRATOS DE SERVICIOS. III.6. LAS SOCIEDADES DE ECONOMÍA MIXTA, ABOCADAS A SER PODERES ADJUDICADORES O ENTES DEL SECTOR PÚBLICO. III.7. AÑADIDOS PRESCINDIBLES: LIMITACIÓN DE LA SÍNTESIS PROCEDIMENTAL A LA LICITACIÓN DEL CONTRATO CONSTITUTIVO Y RÉGIMEN DE MODIFICACIÓN. IV. CONCLUSIONES.

\section{INTRODUCCIÓN: LA IRRUPCIÓN DE LA SOCIEDAD DE ECONOMÍA MIXTA EN LA LEGISLACIÓN CONTRACTUAL}

Hasta que el texto refundido de la Ley de Contratos de las Administraciones Públicas, aprobado por el Real Decreto legislativo 2/2000 fue modificado por la Ley 57/2003, 16 de diciembre, la regulación de las sociedades de economía mixta se había hecho en sede de régimen local. Fue, como se recordará, la referida Ley de medidas de modernización del gobierno local la que trasladó la enumeración de las modalidades de gestión indirecta de los servicios públicos desde el art. 85 de la Ley 7/1995 a la mencionada Ley de Contratos de las Administraciones Públicas, para completar la regulación del contrato de gestión de servicios públicos. Hasta entonces toda la preocupación sobre la licitación concerniente a la constitución de una sociedad de economía mixta se había regulado a través del art. 104.1 del Real Decreto Legislativo 781/1986, de disposiciones legales vigentes de régimen local, con el que continuó conviviendo: «En todo

1 orcid.org/0000-0003-1035-952X. 
caso, deberá determinarse si la participación de los particulares ha de obtenerse únicamente por suscripción de acciones, participaciones o aportaciones de la empresa que se constituya o previo concurso en que los concursantes formulen propuestas respecto a la cooperación municipal y a la particular en la futura Sociedad, fijando el modo de constituir el capital social y la participación que se reserve la Entidad local en la dirección de la Sociedad y en sus posibles beneficios o pérdidas y demás particulares que figuren en la convocatoria».

Pronto se puso de relieve que las exigencias de licitación pública para la adjudicación del contrato de gestión de servicio público no podían ser obviadas a la hora de crear una sociedad de economía mixta, concebida, siguiendo la definición heredada de la legislación local, como aquélla «en la que la Administración participe, por sí o por medio de una entidad pública, en concurrencia con personas naturales o jurídicas» para la prestación del servicio público en cuestión. Y con ello resultó patente que tener que pasar por una doble licitación pública, una primera para seleccionar a los socios privados y otra posterior para adjudicar el contrato de gestión de servicios públicos, no resultaba práctico en la vida real. Era evidente lo desincentivador de una doble licitación para la creación de una sociedad de economía mixta como fórmula de colaboración público-privada, tanto por la dilación inherente a el desarrollo de dichos procedimientos como por la incertidumbre jurídica que existiría en cuanto a la adjudicación de la concesión de gestión de servicios a la sociedad de economía mixta formada con el socio privado previamente seleccionado.

Buscando una solución a la barrera jurídica que representaba esa doble licitación, en su célebre Comunicación interpretativa relativa a la aplicación del Derecho comunitario en materia de contratación pública y concesiones a la colaboración público-privada institucionalizada², la Comisión Europea sugirió sintetizar ambos procedimientos en uno que, respetando el carácter público, transparente y competitivo, pudiera servir para seleccionar el socio privado de una sociedad mixta y adjudicarle el contrato público. Con ello la viabilidad de la operación quedaría garantizada, pues se salvaguardarían los principios fundamentales de los arts. 43 y 49 del Tratado CE, así, como en su caso, de las Directivas sobre contratación pública ${ }^{3}$.

Esta síntesis procedimental fue respaldada por la jurisprudencia del Tribunal de Justicia de la Unión Europea a raíz de su Sentencia de 15 de octubre de 2009 (caso Acoset) ${ }^{4}$, que admitió que la adjudicación directa a la empresa de economía mixta es válida siempre y cuando la selección del socio se haga mediante licitación con pública concurrencia y en condiciones de no discriminación e igualdad de trato, en la que se verifique no sólo la capacidad de aportar el capital sino también los requisitos financieros, técnicos, operativos y de gestión relativos al servicio que deba prestarse y de las características de la oferta en cuanto a las prestaciones que deban realizarse.

Estos son los orígenes de la DA trigésima quinta de la Ley 30/2007, de Contratos de del Sector Público, que fue añadida por la Ley 2/2011, de 4 de marzo, de Economía Sostenible. En su literalidad decía:

«Los contratos públicos y concesiones podrán adjudicarse directamente a una sociedad de economía mixta en la que concurra capital público y privado, siempre que la elección del socio privado se haya efectuado de conformidad con las normas establecidas en la Ley 30/2007, de 30 de octubre, de Contratos del Sector Público para la adjudicación del contrato cuya ejecución constituya su objeto y siempre que no se introduzcan modificaciones en el objeto y las condiciones del contrato que se tuvieron en cuenta en la selección del socio privado.».

Esta síntesis procedimental incorporada nuestro Derecho no es fruto, como se ha visto, de la obligada trasposición de una Directiva, sino de la voluntad de legislador de hacer suya una flexibilización procedimen-

\footnotetext{
2 Véase su apartado 2.2 in fine.

${ }^{3}$ Si bien el contrato de gestión de servicios públicos no resultaba afectado por las Directiva 18/2004 sobre coordinación de los procedimientos de adjudicación de los contratos públicos de obras, de suministro y de servicios, su objeto sí se recoge en los que regula de la Directiva 2014/23/UE relativa a la adjudicación de contratos de concesión.

4 La Sentencia de 15 de octubre de 2009 (asunto C196/08, caso Acoset) se resuelve la cuestión prejudicial planteada por un órgano jurisdiccional italiano planteada en los siguientes términos «¿Es compatible con el Derecho comunitario, en particular con las obligaciones de transparencia y de libre competencia recogidas en los artículos 43, 49 y 86 del Tratado, un modelo de sociedad de economía mixta, creada únicamente para la prestación de un servicio público de naturaleza industrial y con objeto social exclusivo, que sea adjudicataria directa del servicio de que se trata y en la que el socio privado, de carácter "industrial" y "operativo", sea seleccionado mediante un procedimiento de licitación pública, previa verificación tanto de los requisitos financieros y técnicos como de los requisitos propiamente operativos y de gestión relativos al servicio que debe proporcionarse y a las prestaciones específicas que han de ejecutarse?» Para un comentario, ver el trabajo de GALLEGO CÓRCOLES: "La gestión de servicios públicos mediante sociedades de economía mixta en la jurisprudencia europea (I) y (II)", en Contratación administrativa práctica: revista de la contratación administrativa y de los contratistas, núms. 105 y 106 respectivamente, 2011.
} 
tal que se había demostrado compatible con el Derecho europeo de contratación pública, pero que no era exigida por él.

El hasta ahora vigente Texto Refundido de Ley de Contratos del Sector Público, aprobado Real Decreto Legislativo 3/2011 (en adelante TRLCSP), mantiene en su art. 277 la definición de sociedad de economía mixta de la Ley de Bases de Régimen Local, y en su DA 29. ${ }^{a}$ la previsión de fórmulas institucionales de colaboración entre el sector público y el sector privado, conservando el texto de su predecesora, y añadiendo un segundo apartado relativo a las formas de financiación, donde se lee:

«2. Sin perjuicio de la posibilidad de utilizar medios de financiación tales como emisión de obligaciones, empréstitos o créditos participativos, las sociedades de economía mixta constituidas para la ejecución de un contrato público previstas en esta disposición adicional podrán:

a) Acudir a ampliaciones de capital, siempre que la nueva estructura del mismo no modifique las condiciones esenciales de la adjudicación salvo que hubiera estado prevista en el contrato.

b) Titulizar los derechos de cobro que ostenten frente a la entidad adjudicadora del contrato cuya ejecución se le encomiende, previa autorización del órgano de contratación, cumpliendo los requisitos previstos en la normativa sobre mercado de valores.».

\section{DIRECTIVAS EUROPEAS DE 2014 Y SOCIEDAD DE ECONOMÍA MIXTA ¿EFECTO DIRECTO, DEBER DE TRANSPOSICIÓN O LIBERTAD LEGISLATIVA?}

La recientemente promulgada Ley 9/2017, de 8 de noviembre, de Contratos del Sector Público, por la que se transponen al ordenamiento jurídico español las Directivas del Parlamento Europeo y del Consejo 2014/23/UE y 2014/24/UE, de 26 de febrero de 2014 trae causa, como es bien sabido, de las nuevas Directivas de contratación pública: la Directiva 2014/24/UE, sobre contratación pública que viene a sustituir a la Directiva 2004/18/CE sobre coordinación de los procedimientos de adjudicación de los contratos públicos de obras, de suministro y de servicios; y la novedosa la Directiva 2014/23/UE, relativa a la adjudicación de contratos de concesión.

El mayor impacto de estas Directivas para el régimen jurídico de las sociedades de economía mixta se concentra en la configuración del contrato público de concesión de servicio y del contrato público de servicio $^{5}$ como figuras contractuales que sustituyen al contrato de gestión de servicios públicos (por lo general, contrato administrativo ${ }^{6}$ ), al que se venía ligando históricamente en nuestro Derecho la figura de la sociedad de economía mixta como una de sus modalidades.

Pasado el plazo de trasposición de las Directivas, el efecto directo que correspondía reconocerles fue analizado en la Recomendación de la Junta Consultiva de Contratación Administrativa, sobre el efecto directo de las nuevas Directivas comunitarias en materia de contratación pública ${ }^{7}$. En este documento no hay ninguna referencia a las peculiaridades contractuales de las sociedades de economía mixta, salvedad hecha, naturalmente, de la necesidad de aplicar la nueva tipificación contractual antes aludida (contrato de servicio y contrato de concesión de servicio). Tampoco se recogen en las Directivas peculiaridades específicas de

\footnotetext{
5 Sobre estas nuevas figuras, además de la Recomendación de la Junta Consultiva de Contratación Administrativa, sobre el efecto directo de las nuevas Directivas comunitarias en materia de contratación pública de 2016, pueden interesar los siguientes trabajos: GIMENO FELIU, J. M., SALA SÁNCHEZ, P., QUINTERO OLIVARES, G., El interés público y su satisfacción con la colaboración público-privada. Fundamentos, equilibrios y seguridad jurídica. Cámara Oficial de Comercio, Industria, Servicios y Navegación de Barcelona. Barcelona. 2017; "Concesión de servicios y transferencia del riesgo" en Contratación administrativa práctica: revista de la contratación administrativa y de los contratistas, núm. 146, 2016, pág. 102; VILLALBA PÉREZ, F. L., "La concesión de servicios, nuevo objeto de regulación del Derecho Comunitario. Directiva 2014/23/UE de 26 de febrero de 2014 relativa a la adjudicación de contratos de concesión" en Revista de Estudios de la Administración Local y Autonómica: Nueva Época, núm. 2, 2014; RAZQUIN LIZARRAGA, J. A.: "La distinción entre contrato de servicios y concesión de servicios en la reciente jurisprudencia comunitaria y su incidencia en el ámbito interno" en Revista Aranzadi Doctrinal, núm. 3, 2012, pág. 171 y ss; GALLEGO CÓRCOLES, I: "Distinción entre el contrato de concesión de servicios y el contrato de servicios (I) y (II), en Contratación administrativa práctica: revista de la contratación administrativa y de los contratistas, núms. 111 y 112, respectivamente, 2011.

${ }^{6}$ No hace falta recordar que, según el art. 276 TRLCSP en el contrato de gestión de servicios públicos los únicos entes del sector público que podían ser sujetos contratantes eran las Administraciones Públicas (contratos administrativos) y las Mutuas (contratos privados)

7 Esta Recomendación fue publicada en el BOE núm. 66, de 17 de marzo de 2016, por Resolución de 16 de marzo de 2016, de la Dirección General del Patrimonio del Estado.
} 
estas sociedades que, por no reunir las características propias de las normas susceptibles de producir efecto directo, tengan que esperar a ser traspuestas a nuestro Derecho interno para su efectiva aplicación.

Por ello, el legislador español, de cara a la redacción de una nueva Ley de Contratos del Sector Público, se encontraba en plena libertad para haber suprimido, mantenido o reformado el régimen de particularidades propias de las sociedades de economía mixta, como fórmula de colaboración público-privada, incluida la propia definición de sociedad de economía mixta contenida en el art. 277 del TRLCSP. Se trataba en puridad de una opción del legislador estatal, pues de él depende hacer suya la síntesis procedimental de adjudicación aceptada (que no impuesta) por las instituciones de la Unión. Y así lo reconoce claramente la exposición de motivos de la nueva Ley, cuando al comentar que se mantiene la fórmula de adjudicación directa de contratos a una sociedad de economía mixta, especifica que ello se hace «siguiendo el criterio recogido por el Tribunal de Justicia de la Unión Europea en la Sentencia 196/08 en el caso ACOSET, y en la Comunicación Interpretativa de la Comisión Europea relativa a la aplicación del derecho comunitario en materia de contratación pública y concesiones a la colaboración público-privada institucionalizada de 5 de febrero de 2008».

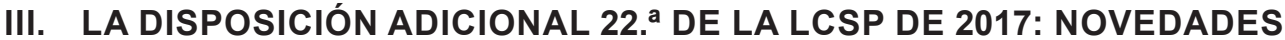 Y OPORTUNIDADES PERDIDAS PARA LAS SOCIEDADES DE ECONOMÍA MIXTA}

En las páginas que siguen se plasman unas primeras reflexiones que acerca del régimen jurídico de las sociedades de economía mixta en la nueva Ley de Contratos del Sector Público de $2017^{8}$, pendiente de publicación al escribir estas líneas.

\section{III.1. ¿Aún en una disposición adicional?}

Como se ha recordado, el régimen procedimental de licitación y adjudicación contractual para las sociedades de economía mixta se introdujo en la LCSP de 2007 en una Disposición Adicional, la 35. ${ }^{\text {a }}$. Desde el punto de vista de técnica legislativa, pese a contar con la excusa de que se trataba de añadir un supuesto concreto a un texto legal en vigor, hacerlo con una disposición adicional ya entonces merecía criticarse. Y lo mismo cabría hacer con el TRLCSP de 2011 donde permaneció como disposición adicional 29. ${ }^{\text {a }}$, cuando bien podría haberse incorporado, con mejor sistemática, al capítulo I del Título I (adjudicación de los contratos) del Libro III de la Ley sobre «selección del contratista y adjudicación de los contratos». Ahora, con mayor razón ha de afearse al legislador que en la nueva Ley haya optado por la comodidad de no evitar la dispersión de preceptos que tratan de los sistemas de adjudicación contractual, en lugar de reunirlos en Capítulo I del Título I del Libro II, junto con las restantes normas de adjudicación de los contratos de las Administraciones Públicas ${ }^{9}$.

Aunque se ha perdido esta oportunidad de mejora sistemática de la LCSP, al menos el cambio de titulación en la nueva Disposición Adicional resalta de forma más clara y directa su contenido. Así, deja de llevar por rótulo «Fórmulas institucionales de colaboración entre el sector público y el sector privado» para pasar a rubricarse «adjudicación de contratos de concesión de obras y de concesión de servicios a sociedades de economía mixta».

\section{III.2. La nueva LCSP altera la definición legal de sociedad de economía mixta}

Al comparar el TRCSP de 2011 con la nueva LCSP pronto se repara en que ésta última no recoge una definición de lo que sea una sociedad de economía mixta, equivalente a la del antiguo art. 277 del texto de 2011. Allí, al hilo de las modalidades de gestión de servicios públicos, se decía que por sociedad de economía mixta se entendía aquélla «en la que la Administración participe, por sí o por medio de una entidad públi$\mathrm{ca}$, en concurrencia con personas naturales o jurídicas». Dado que la nueva Ley da al traste con el contrato de gestión de servicio público y sus distintas modalidades, también lo hace con la noción legal de sociedad de economía mixta. Y esa desaparición tiene consecuencias jurídicas, porque naturalmente, la definición del mencionado 277 no podía desconocerse a la hora de interpretar la DA 29. ${ }^{a}$ TRLCSP y su extensión: cuando

\footnotetext{
${ }^{8}$ Boletín Oficial de las Cortes Generales (Congreso de los Diputados) de 26 de octubre de 2017.

9 Como se sabe, estas son las normas que se toman como referencia a la hora de determinar el régimen de adjudicación de los contratos que celebren los entes del sector público que no sean Administración Pública (arts. 317 y 318 LCSP).
} 
en ella se aludía a la forma de adjudicar contratos a la sociedad de economía mixta, el concepto legal de «sociedad de economía mixta» lo proporcionaba la propia Ley en su art. 277: una sociedad en donde junto a un socio privado participaba otro sujeto merecedor de la condición de «Administración Pública». La conjunción de los dos antiguos preceptos tenía un efecto restrictivo sobre el alcance de la DA 29, que ahora deja de existir. Como veremos, ello potencia de forma importante, e incluso conveniente, considero, la figura de la sociedad de economía mixta.

\section{III.3. La sociedad de economía mixta puede crearse por cualquier ente del sector público, no solo por Administraciones Públicas}

Continuando con el análisis sustantivo de la nueva Ley, hay que destacar que ahora el concepto de sociedad de economía mixta hay que extraerlo de una regulación que, aparentemente, es solamente procedimental. En el nuevo texto, la sociedad de economía mixta se configura sobre la base simplemente de la concurrencia de "capital público» con "capital privado», al margen, se diría, de cualquier especificación de corte subjetivo. Con ello se plantea el interrogante de si la LCSP abandona la exigencia de que el capital público de la sociedad de economía mixta provenga de una Administración pública.

La nueva redacción omite la referencia expresa sobre qué tipo de ente del sector público puede ser adjudicador del contrato a la sociedad de economía mixta. Como los contratos concesión de obra y concesión de servicios, que son los mencionados en la DA 22, no son monopolio de las Administraciones Públicas (sí lo eran los contratos de gestión de servicio público bajo la modalidad aquí analizada ${ }^{10}$ ), puede parecer que el recurso a la sociedad de economía mixta está a la mano de cualquier ente del sector público del art. 3 (aunque no sea Administración Pública).

Sin embargo, antes de reafirmarnos en esta conclusión que hay que preguntarse si realmente cualquier ente del sector público puede aportar ese «capital público» que, unido al «capital privado», caracteriza ahora la sociedad de economía mixta. ¿Puede considerarse público el capital que aporte una fundación pública que lo sea porque, aunque su patrimonio no haya sido aportado por ningún ente público, la mayoría de derechos de voto en su patronato corresponda a representantes del sector público (art. 3.1.e.3. ${ }^{\circ}$ LCSP)? ¿Es capital público el que pueda aportar una Entidad Pública Empresarial estatal, que ha de financiarse mayoritariamente con ingresos de mercado (art. 107. 3 40/2015)?. ¿Lo es, en general, el capital que aporte una entidad con personalidad jurídica propia creada específicamente para satisfacer necesidades de interés general que no tenga carácter industrial o mercantil, cuya actividad no se financie mayoritariamente por poderes adjudicadores, pero que éstos controlen su gestión o nombren a más de la mitad de los miembros de su órgano de administración, dirección o vigilancia (art. 3.3.e LCSP)?.

Mientras que la nueva Ley deja en la indefinición la procedencia de la participación del «capital público», no hace lo mismo cuando en su art. 3.1.h trata de concretar lo que haya de entenderse por sociedad mercantil que es ente del sector público por participación mayoritaria. Ahí sí se desciende a aclarar a tal efecto, que la participación en el capital social en cantidad superior al $50 \%$ debe hacerse por los sujetos mencionados en «los apartados a), b), c), d), e), g) y h)», en donde el g) precisamente alude a las Entidades Públicas empresariales, y el e) a las fundaciones públicas (en cualquiera de sus acepciones). Sería una contradicción que una sociedad mercantil del sector público pudiera originarse por entidades cuyo capital no ha de considerarse público y esas mismas entidades no pudieran participar como parte pública en una sociedad de economía mixta. Esto permite al exégeta de la Ley interpretar en sentido lato la expresión «capital público» que emplea la DA 22. ${ }^{a}$, equivalente a un capital con el origen, al menos, coincidente con el del art. 3.1.h de la nueva Ley.

Por otro lado, para valorar debidamente el calado de la modificación de corte subjetivo introducida por la nueva Ley, recordemos que el art. 277 TRLCSP precisaba que la participación de la Administración en la sociedad de economía mixta podía realizarse «por sí o por medio de una entidad pública». La alusión a aportaciones hechas por una «entidad pública», imposibilitaba la participación en la sociedad de economía mixta de entidades de personalidad jurídico-privada, cosa que parece que puede tenerse por superada en los términos antes expuestos. Cabría, incluso ir más allá, y en puridad, plantear la misma solución respecto a

10 Originariamente el contrato de gestión de servicio público siempre tenía como contratista a una Administración Pública, hasta que la Ley $2 / 2011$, de 4 de marzo lo modificó introduciendo la posibilidad de que, además, pudiera serlo una Mutua de Accidentes de Trabajo y Enfermedades Profesionales de la Seguridad Social. Con todo, la sociedad de economía mixta, como modalidad del contrato de gestión de servicio público, seguía siendo monopolio de la Administración Pública, pues su definición no se vió afectada por la Ley $2 / 2011$. 
los fondos sin personalidad jurídica ${ }^{11}$ (art. 3.1.i LCSP), que no pueden tenerse como entes en sentido estricto por su caracterizadora falta de personalidad, pero que, desde luego, representan un supuesto de «capital público».

\section{III.4. La fórmula de colaboración público-privada de economía mixta sigue restringida a «sociedades»}

No hay novedad en la LCSP acerca de qué sujetos pueden ser contratados con la síntesis procedimental prevista en su DA 22: siguen identificándose como "sociedades» de economía mixta, esto es, entes de personalidad jurídica propia y privada bajo fórmula societaria, que se cualifican, como hemos visto, por la composición híbrida de su capital.

Puede concluirse, por tanto, que los entes personificados bajo formas no societarias siguen quedando al margen de la fórmula de adjudicación que nos ocupa. Sería el caso de otros sujetos jurídico-privados, como puedan ser las fundaciones (que no tienen «capital» en sentido estricto), con el consiguiente resultado de que la LCSP no ampara la aplicación de la síntesis procedimental de adjudicación a la constitución y contratación de una "fundación de economía mixta» ${ }^{12}$. Me pregunto si esto es un resultado querido por el legislador o meramente heredado de normas anteriores por falta de atención durante el procedimiento legislativo, pues no se alcanza razón alguna para excluir de la DA 22 LCSP a estas otras modalidades de colaboración público-privada ${ }^{13}$.

Tampoco se ha preocupado el legislador, como hubiera sido deseable, de especificar el tipo de sociedad que ha de ser una sociedad de economía mixta. La nueva Ley sigue dejando esta cuestión a la interpretación del legislador ¿únicamente puede aplicarse válidamente a las fórmulas societarias de capital, es decir, a sociedades limitadas, sociedades anónimas o sociedades comanditarias por acciones ${ }^{14}$ (estas dos últimas en su modalidad pura o en la de Sociedad Anónima Laboral, Sociedad de Responsabilidad Limitada Laboral, o Sociedad Limitada Laboral $\left.{ }^{15}\right)$ ? ¿O ampara la LCSP además su aplicación, por ejemplo, a las sociedades cooperativas? ${ }^{16}$.

\section{III.5. Variaciones sobre el objeto de la sociedad de economía mixta: se dejan fuera los antiguos contratos de gestión de servicios públicos que ahora son contratos de servicios}

La mayor novedad operada por la LCSP en la DA 22 se refiere a los tipos contractuales para los que se admite la adjudicación directa de contratos a sociedades de economía mixta. Una vez que el objeto del antiguo contrato de gestión de servicios públicos se ha reconducido a los contratos de concesión de servicio y de contrato de servicios ${ }^{17}$, era de esperar que ambos contratos pudieran beneficiarse de la síntesis procedimental de la DA. Es más, desconectada la noción de sociedad de economía mixta del concepto de gestión de servicios públicos ninguna razón habría siquiera para discriminar, en función del objeto del contrato, los supuestos en que poder hacer uso de la adjudicación directa a favor de sociedades de economía mixta. Dicho de otra manera, la desaparición del contrato de gestión de servicios públicos y de sus distintas modalidades como efecto de la Directiva de concesiones parecía sugerir que cobraría plenitud la dicción de

\footnotetext{
11 Sobre el particular, véase CASADO ROBLEDO, S: "El régimen jurídico de los fondos carentes de personalidad jurídica cuya dotación se efectúa mayoritariamente desde los Presupuestos Generales del Estado" en Revista Española de Control Externo, vol. 16, núm. 47, 2014, pág. 83 y ss.

${ }_{12}$ Adviértase, como aclaración, que sí se subsumen en el régimen de la DA 22 LCSP las sociedades de economía mixta cuyas acciones o aportaciones sean de la titularidad de fundaciones, ya públicas ya privadas.

${ }_{13}$ Los fines que, de acuerdo con la Ley de Fundaciones, han de desempeñar estas entidades, son uno de los factores que podrían propiciar estas fundaciones mixtas.

${ }_{14}$ Para la tipología de sociedades de capital, véase el art. 1 del Real Decreto Legislativo 1/2010, de 2 de julio, por el que se aprueba el texto refundido de la Ley de Sociedades de Capital.

15 Todo ello de acuerdo con la Ley 44/2015, de 14 de octubre, de Sociedades Laborales y Participadas.

${ }^{16}$ Sobre esta cuestión véase REGO BLANCO, M. D.: "La cooperativa de servicios públicos en Andalucía como modalidad de sociedad de economía mixta local" en Revista Andaluza de Administración Pública, núm 99, 2017.

17 La trasposición de la Directiva de concesiones no ha estado exenta de sugerencias y críticas, como son las realizadas por MARTÍNEZ-ALONSO CAMPS, J. L.: "El proyecto de ley de contratos del sector público de noviembre de 2016 y la gestión contractual de servicios públicos: análisis de su planteamiento y propuesta alternativa" en Contratación administrativa práctica: revista de la contratación administrativa y de los contratistas, núm. 149, 2017; y por HERNÁNDEZ GONZÁLEZ, F. (2016): “La controvertida supresión del contrato de gestión de servicios públicos" en Revista El Cronista del Estado Social y Democrático de Derecho, núm. 60.
} 
la antigua DA 29. ${ }^{a}$ que se refería a que "los contratos públicos [todos ellos] y las concesiones podrán adjudicarse directamente a una sociedad de economía mixta (...) siempre que (...)". Podría esperarse, pues, que la nueva Ley ampliara las facilidades procedimentales de adjudicación a las sociedades de economía mixta a cualquier contrato público y así aprovechar, cuando conviniera, las ventajas de la colaboración públicoprivada ínsita en las sociedades de economía mixta. Pero no ha sido así.

La LCSP ha beneficiado con la adjudicación directa a dos únicos tipos contractuales, que tienen en común el carácter concesional: concesiones de obras y concesiones de servicios ¿cuál es la razón de esa selección? ¿acaso el carácter concesional, es decir, la transferencia del riesgo operacional al contratista? Si esa fuera la razón, he de decir que no entiende qué vis atractiva existe entre el recurso a una sociedad de economía mixta como contratista y la configuración del contrato con transferencia del riesgo al contratista. Además, si bajo el TRLCSP el contrato de gestión de servicios públicos, cuna de la sociedad de economía mixta, podía cristalizar, según los casos, tanto en contratos que transfirieran el riesgo al contratista como en contratos en que no se transfería ¿cómo justificar ahora que la DA 22 no admita la adjudicación directa de contratos de servicios a sociedades de economía mixta?

\section{III.6. Las sociedades de economía mixta, abocadas a ser poderes adjudicadores o entes del sector público}

Una modificación más incorporada por la DA 22 LCSP se refiere, indirectamente, a la naturaleza jurídico-contractual de la sociedad de economía mixta. La novedad se introduce al regular el aspecto cuantitativo de la participación pública del capital de la sociedad de economía mixta: ahora la LCSP exige que el capital público sea mayoritariamente público (al menos un $51 \%$, por tanto) para que pueda adjudicársele directamente el contrato público de que se trate. Ciertamente, la LCSP no obliga taxativamente a que toda sociedad de economía mixta tenga capital público mayoritario, pero su DA 22, aboca a ello. Esto es evidente teniendo en cuenta que la propia Comisión Europea ha señalado la enorme dificultad que la doble licitación (una para seleccionar socios y otra para adjudicar el contrato) supone para esta fórmula de colaboración público-privada. Por eso, poco o nulo resultado se obtendrá en ese sentido de no aplicar la síntesis procedimental que plasma nuestra DA, pero este procedimiento sintético de licitación, como decimos, la Ley lo reserva, con carácter básico, a las sociedades de economía mixta de capital mayoritariamente público.

Consecuentemente, con la DA 22 LCSP queda anunciado el arrinconamiento, por no decir la desaparición, de las sociedades de economía mixta cuyo capital público sea equivalente o inferior al $50 \%$ del capital social para la ejecución de contratos públicos, cosa que no es fácil de digerir sin una explicación que lo justifique. Y es aquí cuando las preguntas quedan sin respuesta: ¿Por qué se añade esta condición en la nueva DA? Nada dice al respecto la exposición de motivos del proyecto; nada tampoco el Consejo de Estado en su informe acerca del anteproyecto de Ley.

El borrador de anteproyecto de Ley de contratos del sector público de marzo de $2015^{18}$ había omitido totalmente el contenido de la DA 29. ${ }^{a}$ del TRLCSP, pero detectada su falta, se corrigió en el Anteproyecto de Ley que fue objeto del Dictamen del Consejo de Estado, en su informe de 10 de marzo de $2016^{19}$, con una dicción que ha pasado íntegramente al texto aprobado por las Cortes Generales. Pese a que la nueva DA suponía el recorte de la tipología de contratos que pueden suscribirse aplicándoles la síntesis procedimental de licitación, y la exigencia de que las sociedades de economía mixta tengan capital mayoritariamente público, el informe del Consejo de Estado renunció a hacer observaciones sobre el contenido de estas novedades. La excusa fue que la Disposición reproducía en su mayor parte el contenido del texto actualmente vigente. Y ciertamente en la nueva DA 22 se reproduce la DA 29 en su mayor parte, pero las diferencias entre ellas dos, aunque se concretan en pocas palabras, no son insignificantes, como hemos apreciado.

En concreto, la exigencia de capital mayoritario público implica consecuencias de distinto alcance que cabe sintetizar en las siguientes: a) minora las posibilidades de la colaboración privada con las Administraciones Públicas a través de sociedades d economía mixta, al obligar que el capital público sea mayoritario, despreciando las ocasiones en que pudiera ser el capital privado el mayoritario; b) aboca a las sociedades

18 Véase el texto publicado en el portal de transparencia, según visita de 18 de octubre de 2017 (http://transparencia.gob.es/ servicios-buscador/contenido/normaelaboracion.htm?id=NormaEV12L0-20164601\&lang=es\&fcAct=2017-09-26T16:40:24.188Z)

19 El número de expediente del informe es el 1116/2015 (Hacienda y Administraciones Públicas). 
de economía mixta a ser, al menos, entes del sector público, o incluso poderes adjudicadores, atrayendo sobre sí legislación contractual para proveerse de bienes y servicios en el marcado; c) dificulta que las sociedades de economía mixta puedan tener otra naturaleza distinta de las sociedades de capital ${ }^{20}$.

\section{III.7. Añadidos prescindibles: limitación de la síntesis procedimental a la licitación del contrato constitutivo y régimen de modificación} que dice:

Comparando la nueva Ley con el TRLCSP se detecta que en el apartado 1 se ha añadido un párrafo,

«La modificación de los contratos de concesión de obras o de concesión de servicios que se adjudiquen directamente según lo establecido en el párrafo anterior, únicamente se podrá realizar de conformidad con lo establecido en la Subsección $4 .^{a}$ de la Sección $3 .^{a}$ del Capítulo I del Título I del Libro Segundo, relativa a la modificación de los contratos.».

La adición no hace otra cosa que reconducir al régimen general de la modificación de contratos la eventual modificación que pudiera necesitarse en el contrato adjudicado a la sociedad de economía mixta. Salta a la vista la innecesariedad de esta puntualización, pues la forma de adjudicación del contrato (que es la peculiaridad que aborda la Ley en esta Disposición) en nada influye en el régimen de su, modificación, como tampoco lo hace en su cumplimiento o extinción, ni en tantos otros aspectos que, con buen criterio, la Ley no reproduce. Se trata, pues, un añadido prescindible.

Por otro lado, se observa que la nueva DA 22 tiene un apartado más que su predecesora, que se ha intercalado como apartado segundo, entre los dos existentes con anterioridad. En él se establece lo siguiente:

«En el caso en que la sociedad de economía mixta pretendiera acceder como concesionaria a otros contratos distintos de los referidos en el apartado 1 anterior, deberá concurrir al correspondiente procedimiento de licitación de conformidad con lo establecido en la presente Ley.».

Tampoco en esta ocasión ha estado especialmente acertado el legislador en la redacción porque ¿cuáles son los contratos distintos a los de concesión de obra o concesión de servicios (que son los referidos en el apartado 1) a que se puede aspirar a acceder como concesionaria? Se diría que ninguno, pues bajo la tipología contractual de la Ley no hay otras contratos distintos de la concesión de obra y la concesión de servicios a los que se pueda acceder como concesionario. La reducción al absurdo, pues, hace abandonar la interpretación de que la norma se refiera a otros tipos contractuales (de servicio, o de suministro, por ejemplo). Ha de tratarse de otros contratos de concesión de obras o de concesión de servicios diferentes de los que se adjudicaron en su día directamente a la sociedad de economía.

Considero que la Ley pretende recalcar que la sociedad de economía mixta es una sociedad de objeto único, siendo dicho objeto la ejecución del contrato que le fue adjudicado directamente, y que por tanto, una vez materializada dicha adjudicación directa, no puede seguir beneficiándose de nuevas adjudicaciones directas, incluso si una determinación de su objeto social más ambiciosa se lo permitiera. Esto no obsta a que la sociedad de economía mixta pueda participar en procedimientos de licitación de nuevos contratos de concesión, tanto se vehiculen a través de una nueva sociedad de economía mixta, como si no. En el primer caso, no hace falta insistir en que la existencia de un procedimiento de licitación de pública concurrencia, legitimaría, sin duda, una nueva adjudicación directa del ulterior contrato, incluso si el adjudicatario fuera una sociedad de economía mixta previamente existente.

Se trata de subrayar que la adjudicación directa regulada en la Disposición Adicional deriva de una síntesis procedimental, y que ha de estar amparada, ad casum, por una previa selección en pública y libre concurrencia del socio con el que se va a constituir la sociedad de economía mixta. En la misma línea de dejar claro que la adjudicación directa no tiene otra repercusión sobre el contrato de referencia, hay que entender el segundo párrafo del apartado 1 de la DA, donde se especifica la sujeción a las normas comunes sobre modificación de los contratos sujetos a la Ley (Subsección $4 .^{a}$ de la Sección $3 .^{a}$ del Capítulo I del Título I del Libro Segundo). El apartado tiene un claro contenido redundante, y no incorpora novedad alguna, en mi opinión.

20 Por ejemplo, en la legislación andaluza de cooperativas se limita la aportación de los Entes Públicos a las cooperativas de gestión de servicios públicos al 49\% del capital social (art. 54.3 14/2011, de Sociedades Cooperativas Andaluzas ). 


\section{CONCLUSIONES}

La nueva Ley de Contratos del Sector Público de 8 de noviembre de 2017 tiene luces y sombras en lo que a las sociedades de economía mixta se refiere. Es de aplaudir que se haya seguido apostando por la síntesis procedimental que representa la adjudicación directa de contratos a las sociedades mixtas constituidas con las prevenciones legales, pero merece una crítica que no se haya aprovechado para ubicar esta especialidad junto a los restantes procedimientos de adjudicación de contratos.

Más importante resulta el "tira y afloja» que sufre la institución de la sociedad de economía mixta: mientras que se amplía los sujetos que, como parte publica, pueden integrarse en la sociedad, se restringe de forma importante las posibilidades de recurrir a esta figura de colaboración público-privada en un doble e injustificado sentido: se exige participación mayoritaria pública para poder beneficiarse de la adjudicación directa del contrato, y además este queda reducido a contratos de concesiones (de obras y de servicios), cuando antes también se ha utilizado la figura para supuestos reconducidos ahora al contrato de servicios.

Lamentablemente, la impresión general es que el legislador tampoco le ha prestado en esta ocasión la atención que la figura merece. Su régimen jurídico aún queda a la espera de una completa regulación. 